\title{
Estado del arte de la teledermatología
}

\author{
State of the art of tele dermatology
}

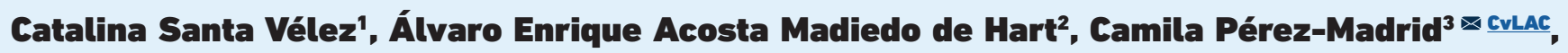 Estefania Galeano Piedrahita“ ${ }^{4}$ CLAC, Daniela Morales Restrepo ${ }^{5}$, Michelle Laasch Restrepo ${ }^{6}$}

Fecha correspondencia:

Recibido: abril 7 de 2020.

Revisado: septiembre 21 de 2020.

Aceptado: octubre 20 de 2020.

Forma de citar:

Santa Vélez C, Acosta Madiedo de Hart AC, Pérez-Madrid C, Galeano Piedrahita E, Morales Restrepo D, Laasch Restrepo M. Estado del arte de la teledermatología. Rev CES Med. 2020; 34(3): 198-206.

Open access

(C) Derecho de autor

Licencia creative commons

Ética de publicaciones

Revisión por pares

Gestión por Open Journal System

DOl: http://dx.doi.org/10.21615/

cesmedicina.34.3.3

ISSN 0120-8705

e-ISSN 2215-9177

Sobre los autores:

1. Dermatóloga oncóloga,

Clínica Vida Fundación, Medellín, Colombia.

Comparte

A0G 소 $R^{\circ}$ 상

\section{Resumen}

El avance de las telecomunicaciones junto con el desarrollo de la fotografía digital han permitido que la Dermatología use herramientas de consulta antes no utilizadas. La teledermatología consiste en la evaluación clínica de lesiones cutáneas por dermatólogos, permitiendo el diagnóstico y el tratamiento a distancia. En este artículo se realiza una revisión de los principales estudios publicados a nivel mundial sobre la teledermatología, diferentes estrategias de aplicación, principales ventajas y desventajas, su legislación y su uso en pandemias.

Palabras clave: Telemedicina; Teledermatología; Dermatología.

\begin{abstract}
The advancement of the telecommunications technologies together with the development of digital photography have allowed dermatology to use previously unexplored consultation tools. Teledermatology consists of the clinical evaluation of skin lesions by dermatologists, to support long-distance clinical health care. This article reviews the main published studies on teledermatology, different strategies of application, advantages and disadvantages of teledermatology, its legislation and its use in pandemics
\end{abstract}

Keywords: Telemedicine, Teledermatology, Dermatology.

\section{Introducción}

La telemedicina es el uso de la tecnología de la telecomunicación para apoyar el cuidado de la salud a distancia. Los avances en la telecomunicación han hecho posible realizar una adecuada atención en el cuidado de la piel cuando el paciente y el médico están separados por tiempo o distancia (1). El modelo de atención de teledermatología ha sido ampliamente descrito. Se usa para atender regiones remotas sin acceso al especialista y para pedir segundas opiniones diagnósticas o terapéuticas de expertos (2).

Se presenta una revisión de la literatura acerca de la teledermatología, la importancia de adicionar teledermatoscopia en el diagnóstico a distancia, la concordancia diagnóstica entre el examen físico presencial y el diagnóstico por teledermatología, la utilidad de la teledermatología como herramienta de clasificación para la práctica clínica, su aceptación por parte del personal médico y pacientes, ventajas y desventajas, su legislación y la teledermatología en tiempos de pandemia. 
2. Dermatólogo oncólogo, Instituto Nacional de Cancerología, Bogotá, Colombia.

3. Dermatóloga, epidemióloga y tricóloga, Universidad CES, Medellín, Colombia.

4. Dermatóloga, Clínica Vida Fundación, Medellín, Colombia.

\section{Residente de} Dermatología, Universidad CES, Medellín, Colombia.

6. Estudiante de Derecho, Universidad EAFIT, Medellín, Colombia.

La teledermatología se ha evaluado en términos de exactitud diagnóstica, resultados clínicos, satisfacción del médico y del paciente, costos, entre otros, demostrando beneficios como aumentar el acceso del paciente a la atención especializada.

\section{Usos}

En Dermatología la toma de decisiones tanto diagnósticas como terapéuticas, está basada en la información clínica obtenida visualmente de la piel del paciente (1). La accesibilidad visual de la piel para la captación y transmisión de un registro fotográfico de enfermedad ha permitido el desarrollo de numerosas aplicaciones dando origen a la teledermatología, facilitado la solución de problemas asistenciales en áreas en las que es baja la disponibilidad de especialistas e incluso dando la posibilidad de poder contar con segundas opiniones en casos de dudas diagnósticas (2,3).

La creciente demanda de servicios dermatológicos, la escasez de especialistas y la falta de acceso a los servicios de salud, resultan en desenlaces clínicos inferiores, reducen la calidad de vida y aumentan los costos de la atención. La evidencia respalda que la teledermatología puede ser un modelo de atención eficaz y rentable (4).

La teledermatología se ha evaluado en términos de exactitud diagnóstica, resultados clínicos, satisfacción del médico y del paciente, costos, entre otros, demostrando beneficios como aumentar el acceso del paciente a la atención especializada, tiempos de espera más cortos, menor número de casos urgentes, correcta evaluación y tratamiento por el dermatólogo e incluso la orientación al médico de atención primaria.

Entre las dermatosis más vistas en los adultos en la teledermatología están las condiciones eccematosas, queratosis seborreicas, enfermedades infecciosas, queratosis actínicas; se encuentra vitiligo, lesiones pigmentadas, cáncer de piel, entre otros. Y en los pacientes pediátricos la dermatitis atópica, molusco contagioso, verruga vulgar, nevus y acné $(5,6)$.

Se han establecido diferentes modelos de teledermatología. Es llamada sincrónica o "en tiempo real" cuando la evaluación se hace a través de videoconferencia; asincrónica cuando se toman fotografías y se envían las imágenes para ser evaluadas por el dermatólogo en un momento posterior; e híbrida cuando se combinan ambas (6).

La modalidad sincrónica requiere el acceso simultáneo a la plataforma. La forma asincrónica emplea una plataforma electrónica para que se envíen imágenes digitales y un historial médico completo al dermatólogo. Esta se prefiere por su rápida accesibilidad, alta utilidad en servicios de salud alejados y por su costo-efectividad $(5,7)$.

Sin embargo, se cuestiona si las evaluaciones remotas de los pacientes son una alternativa clínicamente eficiente. Un estudio retrospectivo compara una cohorte de 11586 pacientes pre-teledermatología y post-teledermatología. Los autores reportan una disminución significativa de los tiempos de atención (84,6 vs 6,7 días), mayor número mensual de paciente evaluados (754 vs 901) y por hora por dermatólogo $(2,27$ vs 2,63$)$. El $61,8 \%$ de los pacientes fueron evaluados sin una visita a la clínica y concluyen que el servicio de dermatología fue el más accesible y eficiente en teledermatología (8).

\section{Importancia de la teledermatoscopia en el diagnóstico de lesiones cutáneas}

El dermatoscopio facilita la visualización de estructuras cutáneas en epidermis, unión dermoepidérmica y dermis papilar, no visibles al ojo desnudo (9). Por lo que la teledermatoscopia ofrece información adicional para mejorar la calidad de las teleconsultas. El uso de la dermatoscopia por examinadores experimentados aumenta 
Se ha observado una concordancia del $74 \%$ entre el examen clínico presencial y el diagnóstico por teledermatología y para la detección del cáncer de piel se ha descrito una sensibilidad del $100 \%$ y especificidad del $90 \%$. la exactitud de diagnóstico de melanoma en un 49 \% en comparación con el diagnóstico clínico al ojo desnudo $(10,11)$. La exactitud de los diagnósticos teledermatoscópicos basados en la histopatología oscila entre el $75 \%$ y el $95 \%$, aumentando la precisión en un $15 \%$ comparado con imágenes clínicas solas (12-14).

Piccolo et al. evalúan la validez de teledermatoscopia en el diagnóstico de melanoma acral y el acuerdo inter-observador de lesiones melanocíticas acrales. Las imágenes de 77 lesiones melanocíticas acrales (71 nevus melanocíticos y seis melanomas) se enviaron a 11 dermatólogos. Todos obtuvieron un alto grado de sensibilidad y especificidad para el diagnóstico, con un acuerdo inter-observador de bueno a excelente (índice kappa 0,40-0,88) para la clasificación de lesiones melanocíticas acrales. Concluyendo que la teledermatoscopia es una herramienta útil para el diagnóstico de melanoma acral y lesiones melanocíticas acrales benignos o potencialmente malignos (15).

La teledermatoscopia mejora la exactitud de los diagnósticos de melanoma en comparación con la foto clínica, aunque también se ha demostrado que continúa siendo inferior al examen persona a persona (3). En un estudio que incluyo 104 lesiones de 80 pacientes, la imagen clínica tuvo mayor concordancia con la histología que la imagen dermatoscópica. En este estudio las tele-evaluaciones clínicas y dermatoscópicas tuvieron una alta concordancia $(K=0,84)$ con el gold estándar y la sensibilidad y especificidad para todas las categorías diagnósticas fueron similares para la foto clínica y teledermatoscópica (16).

\section{Concordancia diagnóstica entre el examen clínico presencial y el diagnóstico por teledermatología/teledermatoscopia}

Se ha observado una concordancia del $74 \%$ entre el examen clínico presencial y el diagnóstico por teledermatología y para la detección del cáncer de piel se ha descrito una sensibilidad del $100 \%$ y especificidad del $90 \%(11,13,17)$.

La concordancia ha sido descrita como excelente para el diagnóstico de melanoma y nevus melanocítico benigno, moderada a muy buena para carcinoma basocelular y moderada a pobre para las queratosis actínicas y el carcinoma escamocelular (18).

En Colombia, Mora et al. valoran la concordancia diagnóstica entre el examen físico realizado por un dermatólogo y el diagnóstico realizado asincrónicamente por otro dermatólogo, encontrando niveles de acuerdo para cáncer de piel de 68-74 \% y para lesiones generales de piel del 51-59\% (7).

\section{La teledermatología como herramienta de triaje}

En un estudio, 20 centros de atención primaria enviaron consultas teledermatoscópicas a dos departamentos de Dermatología para la clasificación de las lesiones cutáneas de interés utilizando una aplicación de teléfono y un dermatoscopio digital. Se comparó el resultado en 816 pacientes remitidos a través de la consulta teledermatoscópica vs 746 pacientes remitidos a través del sistema tradicional. Cuando se requirió tratamiento quirúrgico, el tiempo de espera fue más corto usando teledermatoscopia para los pacientes con melanoma, carcinoma escamocelular y carcinoma basocelular. Las decisiones del triaje también eran más fiables con teledermatoscopia y más del $40 \%$ de los pacientes de teledermatoscopia podrían haber evitado las visitas cara a cara (19). 
En Francia se ha propuesto la teledermatoscopia como estrategia de tamizaje en población rural para el diagnóstico de cáncer de piel (20) y también para el monitoreo de lunares en pacientes pediátricos (21).

Se ha reportado la disminución del tiempo de espera (de 114 días a 39 días), un ahorro de costos del $14 \%$ (22) y se ha demostrado que los pacientes toman fotos dermatoscópicas de calidad suficiente, siempre y cuando se explique con instrucciones por escrito cómo debe realizarse (23).

El diagnóstico mejora al combinar la teledermatología con la teledermatoscopia, pasando de 89 a 91,5 \% cuando se realizan en conjunto (24). Incluso se ha descrito monitoría en casa, a través de la teledermatoscopia por el celular. Sin embargo, para los pacientes con síndrome de nevus displásico, se hace más difícil, ya que sólo evalua una única lesión y no se puede comparar con otras lesiones pigmentadas que presentan los pacientes (25).

Un estudio compara el autoexamen de piel más teledermatoscopia vs el examen clínico completo de piel. Se observó que 22 \% de los pacientes no fotografiaron lesiones pigmentadas que los dermatólogos consideraron requerían monitoreo, la sensibilidad para la identificación de lesiones fue de $42 \%$. En este estudio hubo una correlación alta $(K=0,90)$ entre el telediagnóstico y el examen clínico (25). Por esto se propone la teledermatoscopia asistida donde se agregan unas instrucciones previas para cada paciente de cómo se debe realizar una adecuada autoevaluación de la piel, para que no se excluyan lesiones en áreas difíciles de ver (26).

El diagnóstico mejora al combinar la teledermatología con la teledermatoscopia.
En cuanto a la aceptación de la teledermatoscopia en el personal de la salud, se encuestó a enfermeras y estas percibían que mejoraba la precisión diagnóstica, con un impacto positivo en su práctica (27).

\section{Ventajas y desventajas}

La teledermatoscopia le puede ofrecer el recurso a los médicos de primer nivel, pudiendo acelerar el proceso en casos prioritarios y disminuir el número de interconsultas (cuadro 1).

Cuadro 1. Principales ventajas y desventajas de la teledermatoscopia

\begin{tabular}{ll}
\hline Ventajas & Desventajas \\
\hline $\begin{array}{l}\text { Diagnóstico y tratamiento de los pacientes a } \\
\text { distancia }\end{array}$ & No reemplaza un examen físico \\
\hline Acceso rápido a una consulta dermatológica & Escasa relación médico-paciente \\
\hline Posibilidad de una segunda opinión & Requiere fotografías de buena calidad \\
\hline Fomenta la investigación & $\begin{array}{l}\text { Imposibilidad de tocar las lesiones con mayor } \\
\text { posibilidad de error diagnóstico }\end{array}$ \\
\hline $\begin{array}{l}\text { Reduce los desplazamientos innecesarios } \\
\text { para los pacientes }\end{array}$ & Equipos costosos \\
\hline
\end{tabular}

*Modificado de la referencia 26. 
Otros obstáculos son la infraestructura poco desarrollada en regiones remotas, registros médicos electrónicos fragmentados, modelo económico incompleto, retos legales y éticos.
Otros obstáculos son la infraestructura poco desarrollada en regiones remotas, registros médicos electrónicos fragmentados, modelo económico incompleto, retos legales y éticos (1). Los médicos de primer nivel también han reportado que les preocupa la curva de aprendizaje para la captura de imágenes, que se pierda calidad al enviarse por correo, el acceso a red móvil, entre otros (28).

La tecnología disponible para teledermatoscopia ha sido revisada (29). En el momento, contamos con tecnologías entre softwares y equipos médicos. El software MelaFind permite el análisis de lesiones pigmentadas en 10 bandas espectrales distintas entre infrarrojo y azul del espectro electromagnético. Molemax, Easyscan y Fotofinder son softwares que permiten evaluar objetivamente los cambios de las lesiones de los pacientes en el tiempo. La microscopía confocal usa una técnica conocida como seccionamiento óptico, que genera imágenes comparables a 5 micras en patología y se considera como "biopsia virtual (1). Sin embargo, hasta el momento no existe un estándar tecnológico de plataformas de atención o de equipos para la captura de imágenes autorizados por una sociedad dermatólogica (4).

\section{Teledermatología en tiempos de pandemia}

La pandemia por el coronavirus SARS-CoV-2 ha forzado a cerrar varios servicios de salud en Colombia, atendiendo únicamente urgencias, pacientes hospitalizados y cirugías dermato-oncológicas.

Múltiples plataformas virtuales se han utilizado para realizar consultas virtuales, incluso WhatsApp se ha vuelto popular para su uso como herramienta en la telemedicina. Servicios que esta provee como enviar imágenes, vídeos, interactuar mediante llamada, vídeollamadas, mensaje de texto o voz, adicionales a su fácil uso, simplicidad y costo-efectividad, permiten prestar servicios de teledermatologia en tiempo real con una adecuada interface entre pacientes y dermatólogos (32).

En el momento actual, Colombia no está preparada para afrontar esta pandemia desde la telemedicina, debido a que existe poco conocimiento acerca del tema tanto en el personal de la salud como en los pacientes. Sin embargo, la teledermatología se considera un sustituto efectivo y más en tiempos de pandemia $(30,31)$.

\section{Legislación colombiana en telemedicina}

El presente estudio encuentra la necesidad de resaltar las implicaciones legales de la telemedicina desde los ámbitos de la responsabilidad civil y penal. Un análisis legislativo de la responsabilidad de los especialistas médicos en la aplicación de la telemedicina implica resaltar las posibles negligencias, deficiencias o imprudencias que se puedan desarrollar en la actividad médica y que en consecuencia le puedan generar un daño al paciente atendido por medios electrónicos; de manera que, resulta importante tener en cuenta los códigos éticos, los protocolos y normas vigentes frente a la Telemedicina.

Colombia, a comparación de otros países, tiene una regulación escasa pero suficiente para darle al especialista una guía legal para prestar la atención de manera remota. Mediante la Resolución 2654 de 2019, el Ministerio de Salud y Protección Social establece entonces los parámetros para la práctica de la telemedicina y la telesalud en el país, entre ellos se incluye: (1) garantizar la autenticidad inviolabilidad, integridad, 
La teledermatología permite el diagnóstico y tratamiento de pacientes a distancia; el objetivo principal es permitir el acceso a pacientes que viven en lugares remotos o que no pueden acceder a una consulta dermatológica. disponibilidad y fiabilidad de los datos; (2) utilizar las técnicas necesarias para evitar la suplantación, alteración, pérdida de confidencialidad y cualquier acceso indebido, fraudulento o no autorizado a la misma; (3) la entrega y posterior firma de un consentimiento informado en que se le indique al paciente el alcance de los riesgos, los beneficios, las responsabilidad, el manejo de la privacidad, los protocolos de comunicación y el funcionamiento de la atención (33).

Para la responsabilidad civil, el último parámetro enunciado resulta de vital importancia pues solo con el consentimiento informado se protege la autonomía individual, en tanto le da al paciente un conocimiento mucho más amplio sobre el tipo de atención a la que se está sometiendo voluntariamente y sus posibles riesgos. Vale la pena resaltar que, si bien el consentimiento informado puede eximir de responsabilidad civil por posibles diagnósticos erróneos que se den por la atención remota, nunca se eximirá la responsabilidad cuando se presente una mal praxis médica. Frente a la responsabilidad penal, es conveniente seguir de manera juiciosa los parámetros de protección de datos personales, pues el Código Penal colombiano en artículo 269 - F tipifica la violación de datos personales y la sanciona con pena privativa de la libertad de cuarenta y ocho meses a noventa y seis meses y multas de 100 a 1000 salarios mínimos legales mensuales vigentes. Las consecuencias jurídicas del incumplimiento de los deberes médicos impuestos en legislación sobre la materia serán cuantiosas indemnizaciones por demandas civiles; penas que pueden ir desde multas hasta penas privativas de la libertad (33).

\section{Conclusiones}

La teledermatología permite el diagnóstico y tratamiento de pacientes a distancia; el objetivo principal es permitir el acceso a pacientes que viven en lugares remotos o que no pueden acceder a una consulta dermatológica. Existen muchas limitaciones, que no superan los beneficios y algunas de ellas pueden ser superadas a través de una evaluación adecuada por el personal de atención primaria. Entre ellas esta la necesidad de una historia clínica completa, fotografías de muy alta calidad. Debido a que es una tecnología emergente, también se debe estudiar la legislación colombiana que regula la telemedicina, en la cual es imperativo el uso del consentimiento informado, en donde el paciente entienda las limitaciones de la atención que se le esta presentando y acepte los posibles riesgos.

\section{Conflictos de intereses}

Los autores declaran no tener conflicto de intereses

\section{Bibliografía}

1. Coates SJ, Kvedar J, Granstein RD. Teledermatology: From historical perspective to emerging techniques of the modern era. J Am Acad Dermatol. 2015;72 (4):577-86.

2. Kanthraj G. Newer insights in teledermatology practice. Indian J Dermatol Venereol Leprol. 2011;77 (3):276.

3. Fabbrocini G, Balato A, Rescigno O, Mariano M, Scalvenzi M, Brunetti B. Telediagnosis and face-to-face diagnosis reliability for melanocytic and non-melanocytic 'pink' lesions. J Eur Acad Dermatol Venereol. 2008;22 (2):229-34 
4. Wang RH, Barbieri JS, Nguyen HP, Stavert R, Forman HP, Bolognia JL, et al. Clinical effectiveness and cost-effectiveness of teledermatology: Where are we now, and what are the barriers to adoption? J Am Acad Dermatol. 2020;S0190962220301511.

5. Naka F, Makkar H, Lu J. Teledermatology: Kids are not just little people. Clin Dermatol. 2017;35 (6):594-600.

6. Eissing L, Trettel A, Augustin M. Telemedicine in dermatology: findings and experiences worldwide - a systematic literature review. J Eur Acad Dermatology Venereol. 2018;32(2):215-44.

7. Mora ÓE, Olmos Olmos E, Rochel Granados CM, Torres Pradilla M, Rodríguez N. Acuerdo entre el examen dermatológico directo y teledermatología asincrónica prueba piloto, hospital de San José, Bogotá DC, Colombia. Rev Repert Med Cir. 2012;21 (2):122-5.

8. Zakaria A, Maurer T, Su G, Amerson E. Impact of teledermatology on the accessibility and efficiency of dermatology care in an urban safety-net hospital: A pre-post analysis. J Am Acad Dermatol. 2019;81 (6):1446-52.

9. Marghoob AA, Usatine RP, Jaimes N. Dermoscopy for the family physician. Am Fam Physician. 2013;88(7):441-50

10. Börve A, Terstappen K, Sandberg C, Paoli J. Mobile teledermoscopy-there's an app for that! Dermatol Pract Concept. 2013;3 (2).

11. Arzberger E, Curiel-Lewandrowski C, Blum A, Chubisov D, Oakley A, Rademaker $M$, et al. Teledermoscopy in high-risk melanoma patients: a comparative study of face-to-face and teledermatology visits. Acta Derm Venereol. 2014;0.

12. Blum A, Hofmann-Wellenhof R, Luedtke H, Ellwanger U, Steins A, Roehm S, et al. Value of the clinical history for different users of dermoscopy compared with results of digital image analysis. J Eur Acad Dermatol Venereol [Internet]. 2004;18 (6):665-9.

13. Piccolo D, Smolle J, Argenziano G, Wolf IH, Braun R, Cerroni L, et al. Teledermoscopy - results of a multicentre study on 43 pigmented skin lesions. J Telemed Telecare. 2000;6 (3):132-7.

14. Provost N, Kopf AW, Rabinvitz HS, Stolz W, DeDavid M, Wasti Q, et al. Comparison of conventional photographs and telephonically transmitted compressed digitized images of melanomas and dysplastic nevi. Dermatology. 1998;196 (3):299-304.

15. Piccolo D, Soyer HP, Chimenti S, Argenziano G, Bartenjev I, Hofmann-Wellenhof $\mathrm{R}$, et al. Diagnosis and categorization of acral melanocytic lesions using teledermoscopy. J Telemed Telecare [Internet]. 2004;10 (6):346-50. 
16. Kroemer S, Frühauf J, Campbell TM, Massone C, Schwantzer G, Soyer HP, et al. Mobile teledermatology for skin tumour screening: diagnostic accuracy of clinical and dermoscopic image tele-evaluation using cellular phones. $\mathrm{Br} J$ Dermatol. 2011;164 (5):973-9.

17. Tan E, Yung A, Jameson M, Oakley A, Rademaker M. Successful triage of patients referred to a skin lesion clinic using teledermoscopy (IMAGE IT trial): Teledermoscopy as triage for skin lesions. Br J Dermatol. 2009;162 (4):803-11

18. Tan E, Oakley A, Soyer HP, Haskett M, Marghoob A, Jameson M, et al. Interobserver variability of teledermoscopy: an international study: Interobserver variability of teledermoscopy. Br J Dermatol [Internet]. 2010;163 (6):1276-81

19. Börve A, Gyllencreutz J, Terstappen K, Backman E, Aldenbratt A, Danielsson M, et al. Smartphone teledermoscopy referrals: A novel process for improved triage of skin cancer patients. Acta Derm Venereol. 2015;95 (2):186-90.

20. Hue L, Makhloufi S, Sall N'Diaye P, Blanchet-Bardon C, Sulimovic L, Pomykala $F$, et al. Real-time mobile teledermoscopy for skin cancer screening targeting an agricultural population: an experiment on 289 patients in France. J Eur Acad Dermatol Venereol. 2016;30 (1):20-4.

21. Cheng H, Oakley A, Rademaker M. Change in a child's naevus prompts referral to a dermatology service. J Prim Health Care. 2014;6 (2):123-8.

22. Lim D, Oakley AM, Rademaker M. Better, sooner, more convenient: A successful teledermoscopy service: Teledermoscopy service. Australas J Dermatol. 2012;53 (1):22-5.

23. Marchetti MA, Fonseca M, Dusza SW, Scope A, Geller AC, Bishop M, et al. Dermatoscopic imaging of skin lesions by high school students: a cross-sectional pilot study. Dermatol Pract Concept. 2015;5 (1):11-28.

24. Massone C, Hofmann-Wellenhof R, Ahlgrimm-Siess V, Gabler G, Ebner C, Soyer HP. Melanoma screening with cellular phones. PloS One. 2007;2 (5):e483.

25. Manahan MN, Soyer HP, Loescher LJ, Horsham C, Vagenas D, Whiteman DC, et al. A pilot trial of mobile, patient-performed teledermoscopy. $\mathrm{Br} \mathrm{J}$ Dermatol. 2015;172 (4):1072-80.

26. Janda M, Loescher LJ, Banan P, Horsham C, Soyer HP. Lesion Selection by Melanoma high-risk consumers during skin self-examination using mobile teledermoscopy. JAMA Dermatol [Internet]. 2014;150 (6):656.

27. Stratton D, Loescher LJ. The acceptance of mobile teledermoscopy by primary care nurse practitioners in the state of Arizona: J Am Assoc Nurse Pract. 2016;28 (6):287-93.

28. Chao JT, Loescher LJ, Soyer HP, Curiel-Lewandrowski C. Barriers to mobile teledermoscopy in primary care. J Am Acad Dermatol. 2013;69 (5):821-4 
29. Ferrara G, Argenziano G, Cerroni L, Cusano F, Di Blasi A, Urso C, et al. A pilot study of a combined dermoscopic-pathological approach to the telediagnosis of melanocytic skin neoplasms. J Telemed Telecare. 2004;10 (1):34-8

30. Villani A, Scalvenzi M, Fabbrocini G. Teledermatology: a useful tool to fight COVID-19. J Dermatolog Treat. 2020.

31. Chawla S. COVID-19: Challenges and opportunities for dermatology response. J Dermatolog Treat. 2020.

32. Jakhar D, Kaul S, Kaur I. WhatsApp messenger as a teledermatology tool during coronavirus disease (COVID-19): From bedside to phone-side. Clin Exp Dermatol. 2020.

33. Resolución 2654 de 2019, Ministerio de Salud y Protección social, Colombia. 